248

IDENTIFICATION OF MAJOR CLINICAL CHARACTERISTICS OF CHINESE SLE PATIENTS AND LINEAR CORRELATIONS AMONG SLEDAI, SF-36 AND HADS-ANXIETY USING MOBILE SMART SYSTEM OF DISEASE MANAGEMENT (SSDM)

${ }^{1} \mathrm{GS}$ Wang, ${ }^{2} \mathrm{~J}$ Yang, ${ }^{3} \mathrm{XW}$ Duan, ${ }^{4} \mathrm{ZB}$ Wu, ${ }^{5} \mathrm{JL}$ Huang, ${ }^{6} \mathrm{JL} \mathrm{Ru},{ }^{7} \mathrm{~T}$ Xie, ${ }^{8} \mathrm{YF}$ Wang, ${ }^{9} \mathrm{C}$ Zhao, ${ }^{10} \mathrm{R} \mathrm{Wu},{ }^{11} \mathrm{H}$ Wei, ${ }^{12} \mathrm{ZC}$ Zhang, ZJ Li $\mathrm{Li}^{13},{ }^{14} \mathrm{HB} \mathrm{Li},{ }^{15} \mathrm{XX}$ Zuo, ${ }^{16} \mathrm{HL} \mathrm{Wu},{ }^{17} \mathrm{YS} \mathrm{Li}$, ${ }^{18} \mathrm{YH}$ Jia, ${ }^{18} \mathrm{~F}$ Xiao, ${ }^{1} \mathrm{XM}$ Li. ${ }^{1}$ Anhui Provincial Hospital, Department of rheumatology, Hefei, China; ${ }^{2}$ Central Hospital of MianYang- Sichuan, Department of rheumatology, MianYang, China; ${ }^{3}$ The Second Affiliated Hospital of Nanchang University-Nanchang, Department of rheumatology, Nanchang, China; ${ }^{4}$ The First Affiliated Hospital of The Fourth Military Medical University, Department of Rheumatology and Immunology, Xi'an, China; ${ }^{5}$ The Sixth Hospital Affiliated to Sun yat-sen University, Department of rheumatology, Guangzhou, China; ${ }^{6}$ The 264th Hospital of the PLA, Department of rheumatology, Taiyuan, China; ${ }^{7}$ Affiliated hospital of Guangdong medical University, Department of rheumatology, Zhanjiang, China; ${ }^{8}$ The First Affiliated Hospital of BaoTou Medical College, Department of rheumatology, Baotou, China; ${ }^{9}$ The First Affiliated Hospital of Guangxi Medical University, Department of rheumatology, Nanning, China; ${ }^{10}$ The First Affiliated Hospital of Nanchang University, Department of rheumatology, Nanchang, China; "Northern Jiangsu People's Hospital, Department of rheumatology, Yangzhou, China; ${ }^{12}$ People's Hospital of Linyi- Shandong, Department of rheumatology, Linyi, China; ${ }^{13}$ The First Affiliated Hospital of Bengbu Medical College, Department of rheumatology, Bengbu, China; ${ }^{14}$ The Affiliated Hospital of Inner Mongolia Medical University, Department of rheumatology, Hohhot, China; ${ }^{15}$ Xiangya Hosipital of Central South University, Department of rheumatology, Changsha, China; ${ }^{16}$ People's Hospital of Dongguan, Department of rheumatology, Dongguan, China; ${ }^{17}$ People's Hospital of Zhejiang Province, Department of rheumatology, Hangzhou, China; ${ }^{18}$ Gothic Internet Technology Corporation, Medical Department, Shanghai, China

10.1136/lupus-2017-000215.248

Background and aims The association among SLEDAI, SF-36 and HADS in China was unknown. Smart System of Disease Management (SSDM) is a series of mobile applications for chronic diseases management. The purpose of this study is to describe major clinical characteristics of Chinese SLE patients using SSDM and analyse the potential association among SLEDAI, SF-36 and HADS.

Methods SSDM includes physicians' and patients' application system. The patient application system includes SLEDAI, SF36, HADS and medication management. After data entry, patients can synchronise data to the mobile terminal of authorised rheumatologist. All patients fulfilling the 1997 ACR criteria for SLE were recruited.

Results A total of 3717 SLE patients from 490 rheumatologists in 214 rheumatology centres across China participated in the study ( $89 \%$ were women). The mean age was $34.09 \pm 11.87$ years and the median disease duration was 3.15 years.1,908 patients performed self-assessment for 3085 times. The mean score of SLEDAI, SF-36, HADS-Anxiety (HADS-A) and HADS-Depression (HADS-D) were 9.41 $\pm 2.52,60.09 \pm 20.01$, $7.86 \pm 4.09$ and $8.77 \pm 4.25$ respectively. According to the SLEDAI criteria, $43.71 \%, 18.50 \%, 13.42 \%$ and $24.37 \%$ patients achieved Remission, Low, Moderate and High disease activity. SLEDAI was significantly correlated with SF-36 and HADS-A independently. The regression equation was "SLEDAI=21.753$0.179 *$ SF-36" $(p=0.011)$ and "SLEDAI $=0.461+1.114 *$ HADSA" $(\mathrm{p}=0.028)$.

Conclusions SSDM is an effective mobile interface to serve for SLE patients performing self-management as well as to supply physicians with valuable data. SLEDAI was significantly correlated with SF-36 and HADS-A independently.

\section{9 LIKELIHOOD OF PATIENTS WITH INCOMPLETE LUPUS TO ENTER A RANDOMISED, PLACEBO-CONTROLLED TRIAL OF HYDROXYCHLOROQUINE}

${ }^{1} \mathrm{D}$ Karp* ${ }^{*}{ }^{2} \mathrm{~B}$ Chong, ${ }^{3} \mathrm{C}$ Arriens, ${ }^{3} \mathrm{~J}$ James, ${ }^{4} \mathrm{M}$ Ishimori, ${ }^{4} \mathrm{M}$ Weisman, ${ }^{4} \mathrm{D}$ Wallace, ${ }^{5} \mathrm{~N}$ Olsen. ${ }^{1}$ UT Southwestern Medical Centre, Rheumatic Diseases Division, Dallas, USA; ${ }^{2}$ UT Southwestern Medical Centre, Dermatology, Dallas, USA; ${ }^{3}$ Oklahoma Medical Research Foundation, Arthritis and Clinical Immunology, Oklahoma City, USA; ${ }^{4}$ Cedars Sinai Medical Centre, Rheumatology, Los Angeles, USA; ${ }^{5}$ Penn State Hershey Medical Centre, Rheumatology, Hershey, USA

\subsection{6/lupus-2017-000215.249}

Background and aims Hydroxychloroquine (HCQ) is used by the majority of patients who have incomplete lupus erythematosus (ILE), defined as positive ANA and 1-2 other criteria for SLE, although efficacy in this situation has never been proven in a rigorous clinical trial. The Study of anti-Malarials in Incomplete Lupus Erythematosus (SMILE) is a proposed placebo-controlled trial of HCQ in ILE designed to measure the effect of drug on progression to SLE. In order to judge trial feasibility, "mock recruitment" was performed.

Methods 45 patients seen in outpatient clinics of the SMILE investigators for ANA and musculoskeletal or cutaneous complaints were interviewed using a structured script explaining the need for the trial, potential risks and benefits of HCQ and the possible randomization to placebo. They were then asked questions to ascertain their understanding of the trial and their willingness to enrol.

Results $96 \%$ of the subjects were female; median age was 35 and median symptom duration 3 years. 13\% were Hispanic and $13 \%$ were African American. $18 \%$ had a personal history of autoimmune disease other than lupus; $42 \%$ had a family history. Musculoskeletal and cutaneous symptoms were each in $60 \%$ of subjects. $73 \%$ of subjects were interested, and $64 \%$ were likely to enrol. The most common reason for disinterest was lack of time to participate $(50 \%)$, risks of HCQ $(25 \%)$ and possibility of getting placebo (19\%).

Conclusions A placebo-controlled clinical trial is feasible when the standard of care is an approved drug. 50\% more subjects need to be screened to have enough to enrol in the trial.

\section{EVALUATION OF ATOPY AND EOSINOPHILIA IN PATIENTS WITH PSORIASIS}

${ }^{1} \mathrm{M}$ Khoshkhuiं, ${ }^{2} \mathrm{P}$ Hosseini, ${ }^{3} \mathrm{M}$ Rezaie, ${ }^{2} \mathrm{R}$ Faridhosseini. ${ }^{1}$ Mashhad, Iran; ${ }^{2}$ Mashhad University Of Medical Science, Allergy and Clinical Immunology, Mashhad, Iran; ${ }^{3}$ Mashhad University Of Medical Science, Cardiology, Mashhad, Iran

\subsection{6/lupus-2017-000215.250}

Background and aims Psoriasis is a TH1 and TH17 cellsdependent autoimmune disease of the skin and joint while allergic disorders are TH2 cell-dependent. There are conflicting reports about the effect of atopy on psoriasis. With regard to these reports, the aim of the current study was to determine the frequency of atopy, allergic disorder (such as allergic rhinitis, asthma and eczema) and eosinophilia in patients with psoriasis

Methods For this purpose, this case-control study was performed in Mashhad Ghaem hospital. History of allergic diseases including: allergic rhinitis, asthma and eczema were evaluated based on ISAAC standard questionire. Skin prick test was performed with 5 common aeroallen in our reigeon and atopy was define as a result of only one positive skin 
prick test.Also peripheral blood samples were obtained to determine serum levels of IgE and blood eosinophil cell count in both study groups.

Results A total of 52 patients with psoriasis, and 50 healthy subjects as the control group were considered. Between allergic diseases only asthma was lower in psoriatic patients rather than controlbased on ISAAC questionarie $(p=0.044)$. There is no significant diffrence between result of skin prick test (atopy) in case and control group(p). Also no significant correlation was found between eosinophil count $(\mathrm{p}=0.057)$ and Ig E level in case and control group $(p=0.88)$.

Conclusions In general, these findings suggest that the Asthma was not common in psoriasis, and support the concept that Asthma protects against the autoimmune disease such as psoriasis.

\section{ASSOCIATED FACTORS ON SLEEP DISORDERS IN SYSTEMIC LUPUS ERYTHEMATOSUS}

${ }^{1} Y$ Li. ${ }^{1}$ Xiangya hospital - Central South University, Department of Rheumatology and Immunology, Chang Sha, China

\subsection{6/lupus-2017-000215.251}

Background and aims To investigate the prevalence of sleep disorders and the relevant determinants in a cohort of SLE patients.

Methods One hundred patients with SLE were included in the study. Sleep quality was assessed using the Pittsburgh Sleep Quality Index (PSQI). Depression, anxiety, quality of life, and fatigue were evaluated by PHQ-9, GAD-7, SF-36, and VAS respectively. The demographic and clinical data were also recorded. SLE disease activity and damage severity were assessed by SLEDAI and SDI respectively.

Results The prevalence of sleep disorders in SLE patients was $42 \%$. Compared with patients without sleep disorders, the ratio of males and married patients, age, the score of SDI, PHQ-9, GAD-7, and fatigue were higher in SLE patients with sleep disorders, while the score of SF-36 was lower $(p<0.05)$. Age, SLEDAI, SDI, PHQ-9, GAD-7, and fatigue correlated positively with sleep disorders $(p<0.05)$. C3 and the score of SF-36 correlated negatively with sleep disorders $(\mathrm{p}<0.05)$. In multiple logistic regression analyses, gender, anxiety, body pain, and energy were the independent determinants of sleep disorders $(\mathrm{R} 2=0.494, \mathrm{P}<0.01)$.

Conclusions Poor sleep quality is common in SLE patients. Gender, age, disease activity and severity, anxiety, depressed mood, and quality of life contribute significantly to sleep disorders in SLE.

\section{THE LONG-TERM OUTCOMES ON LEFLUNOMIDE TREATMENT IN PATIENTS WITH LUPUS NEPHRITIS: A TWELVE-YEAR FOLLOW-UP STUDY}

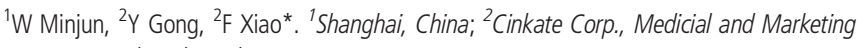
Department, Shanghai, China

\subsection{6/lupus-2017-000215.252}

Background and aims One phase III study has reported that Leflunomide(LEF) in combination with prednisone was effective in the induction therapy of proliferative lupus nephritis (LN). However, long-term data of LEF in the maintenance treatment of $\mathrm{LN}$ are lacking. The aim of this study was to investigate the long-term outcomes of LEF in the maintenance treatment of LN in an open-label extension of a prospective multi-centre observational study.

Methods 70 patients were enrolled into LEF induction group with a loading dose of $50 \mathrm{mg} /$ day for 3 days, followed by $30 \mathrm{mg} /$ day for six months. The partial and complete remission rate was $52 \%$ and $21 \%$. Among them, 15 patients voluntarily entered an open labelled extension study. Data for survival and kidney function were prospectively collected during a 12 year period for the 15 patients who were maintained on $20 \mathrm{mg} /$ day LEF.

Results 15 patients (mean age $40.2 \pm 7.4$ years; $86.6 \%$ female) were treated with LEF for $>12$ months, the mean duration of LEF treatment was 11.8 years. During a median follow-up period of 12.3 years, 1 patient died from sepsis, 4 patients developed renal flare. The 12 year cumulative survival rates for the composite end point of death and chronic renal failure were $93.3 \%$ and $100 \%$, respectively. At the final follow-up, the complete remission rate in the remaining patients was $71.43 \%$, the partial remission rate was $28.57 \%$, the mean proteinuria leval was $0.33 \mathrm{~g} /$ day(range $0.07-1.62 \mathrm{~g} /$ day), and the mean serum creatinine leval was $62.3 \mathrm{umol} / \mathrm{L}$.

Conclusions Our data suggest that a remission-inducing regimen of LEF followed by maintenance therapy with LEF resulted in good long-term patient survival and renal preservation.

\section{A COMPARISON OF OVARIAN RESERVE IN WOMEN WITH SYSTEMIC LUPUS ERYTHEMATOSUS FOLLOWING TREATMENT WITH INTRAVENOUS CYCLOPHOSPHAMIDE VIS ORAL MYCOPHENOLATE MOFETIL}

${ }^{1} \mathrm{P}$ Potturi*, ${ }^{1} \mathrm{~S}$ Sharma, ${ }^{2} \mathrm{P}$ Sikka, ${ }^{3} \mathrm{M}$ Rathi, ${ }^{4} \mathrm{~N}$ Sachdeva, ${ }^{1} \mathrm{~S}$ Jain. ${ }^{1} \mathrm{PGIMER}$, Internal Medicine, Chandigarh, India; ${ }^{2} P G I M E R$, Gynaecology and Obstetrics, Chandigarh, India; ${ }^{3}$ PGIMER, Nephrology, Chandigarh, India; ${ }^{4}$ PGIMER, Endocrinology, Chandigarh, India

\subsection{6/lupus-2017-000215.253}

Introduction Immunosuppression with Cyclophosphamide is treatment of choice in lupus nephritis. Ovarian reserve depletion is well known complication. Early identification of depletion of ovarian reserve can be done by correlating antral follicle count with AMH.

Aims- To assess the ovarian reserve in patients who have receive d IV Cyclophosphamide vs those who have received Mycophenolate-mofetil for SLE by measuring $\mathrm{AMH}$, Inhibin $\mathrm{B}, \mathrm{FSH}$ and antral follicular count.

Methods A prospective Case-Control study with 50 patients who were diagnosed with SLE were studied. Twenty-five cases and controls defined by females age 18-40 with SLE were enrolled. Cases received IV CYC as per NIH protocol and controls received oral MMF. Baseline FSH, LH, E2, AMH, Inhibin and antral follicular count, and at 6 months were done in both the groups.

Results Mean age: cases- 31.36 \pm 6.357 , controls- $28.36 \pm 4.396$ (p-0.058).

Conclusions MMF did not cause decrease in ovarian reserve. IV CYC caused Subclinical depletion of ovarian reserve with significant decreased in $\mathrm{AMH}$, Inhibin B and E2 levels and follicular counts. AMH and AFC are good markers to assess subclinical depletion of ovarian reserve 